\title{
Quantum interferometry, entanglement, and holography
}

\author{
Bahaa E. A. Saleh*, Ayman F. Abouraddy, Alexander V. Sergienko, \\ and Malvin C. Teich \\ Quantum Imaging Laboratory, Department of Electrical \& Computer Engineering, \\ Boston University, Boston, MA 02215, USA
}

\begin{abstract}
Entangled-photon beams, generated for example by spontaneous parametric down-conversion from a nonlinear crystal, may be used to implement holography based on quantum interferometry. One beam is split into a probe component that illuminates the object and another serving as a reference, and is detected with no spatial resolution. The other beam is measured with full spatial resolution. The rate of two-photon coincidence contains full holographic information on the object. Since the beams are not monochromatic, this is a form of higher-order white light holography.
\end{abstract}

\section{INTRODUCTION}

Classical optical interferometry, a ubiquitous tool in modern optics, has applications ranging from metrology to holography. Interferograms are often used to measure the spatial and temporal coherence of optical fields, which may carry information on the light source. Classical higher-order coherence, as measured by intensity interferometers, has also found applications such as the measurement of the angular diameter of stars. In recent years, two-photon interferometry has come into vogue as the generation of optical beams in two-photon quantum states has become feasible via the process of spontaneous parametric down conversion in nonlinear crystals $^{1}$. A number of topologies for creating the indistinguishable paths necessary for quantum interferometry have been demonstrated, and some applications have been considered, including metrology, imaging, secure communication and cryptography.

Entanglement is a key attribute that the quantum two-particle state may be endowed with. While entanglement is not necessary for two-photon interferometry, it is a fundamental requirement for certain applications, such as two-photon coherent imaging ${ }^{2}$. In this application, one of the two entangled beams probes a remote object, while the other is detected locally. Appropriate measurement of the rate of two-photon coincidence may be used to reconstruct a coherent image of the object, even though the probe beam is, by itself, incoherent. It is the higher-order coherence associated with entanglement that enables coherent imaging. Entanglement is a requirement for this remarkable feature of two-photon coherent imaging. As will be shown in this paper, entanglement may offer access to information that cannot be measured by any classical means. Thus, entangled-based quantum imaging transcends the capabilities of classical imaging.

\section{QUANTUM HOLOGRAPHY}

The possibility of entangled-photon coherent imaging suggests entangled-photon holography. Conventional holography is based on the interference of an optical wave, scattered from a 3-D object, with a reference wave generated from the same source. Likewise, two-photon interferometry may be used in holography. One configuration (Fig. 1) uses, as a source, two beams of light in a two-photon entangled state generated, for example, by spontaneous parametric down conversion from a nonlinear crystal. One beam (signal) is split into two components: the object beam, which is scattered from the object, and the reference. Both components of the signal beam overlap at the detector (D1). The idler beam is measured locally with detector (D2) without interacting with the object. The signal beam would, if it were coherent, create a hologram on detector D1 based on conventional interference between the reference and the probe beams. Being incoherent and broadband, however, this beam cannot produce a stable interference pattern. Nevertheless, because of the underlying entanglement, appropriate combined measurements of the idler and signal beams can yield holographic information.

Assuming full spatial entanglement, it has been shown ${ }^{3}$ that the coincidence rate $C\left(x_{1}, x_{2}\right)$, i.e., the probability density of detecting one photon at position $x_{1}$ in the signal beam and another at position $x_{2}$ in the idler beam, contains a discernable term identical to that generated in conventional holography. More remarkably, full holographic information is encoded in the probability density $p\left(x_{2}\right)$ of detecting one photon at position $x_{2}$ in the idler beam, given that a photon is observed at any point $x_{1}$ in the signal beam. The interference 
term in $p\left(x_{2}\right)$ turns out to be proportional to a coherent image of the object formed through an optical path beginning from the object, advancing toward detector D1 and reflecting therefrom, retracing the reference beam toward the source $\mathrm{S}$ and reflecting therefrom, and finally retracing the idler beam back to detector D2. Optical components may be inserted in the idler beam to manipulate that image, as desired. The function $p\left(x_{2}\right)$ is recorded by local measurement of the photon arrival rate as a function of position $x_{2}$ in the idler beam using a detector D2 gated by the arrival of a signal photon anywhere on the surface of detector D1. The function $p\left(x_{2}\right)$ may be measured by a large collection of two-photon registrations, observed over a long period of time. This function may subsequently be recorded on a 2-D photographic plate or spatial light modulator, and viewed with ordinary light in the usual fashion of holographic reconstruction.

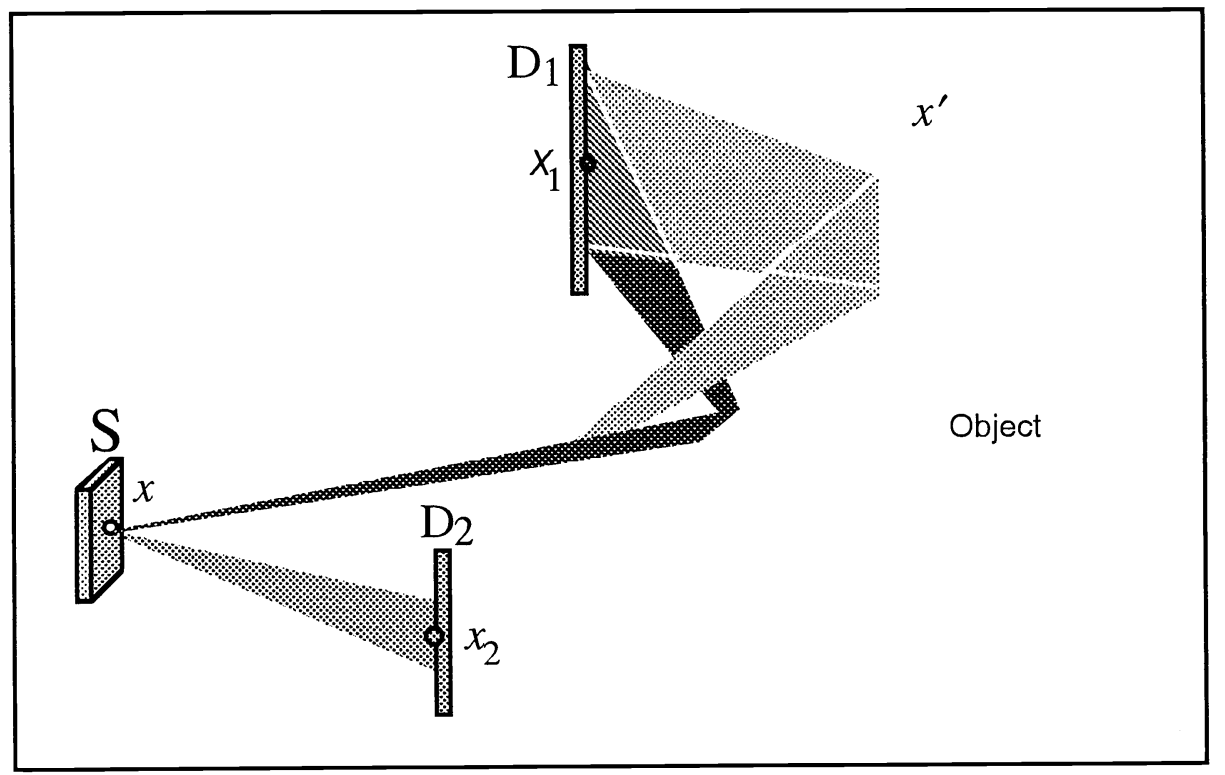

Fig. 1. Quantum holography. A source $S$ generates two beams in an entangled-photon state, signal and idler. The signal beam is split into a probe beam, which scatters from the object, and a reference beam which mixes with the scattered beam at detector D1, The idler beam is detected with detector D2, which has full spatial resolution. Detector D1 has no spatial resolving power.

\section{CONCLUSION}

The fact that the signal beam, which probes the object in a two-path configuration, is observed with no spatial resolution is also remarkable. No classical beam is capable of extracting this type of 3-D spatial information with a single integrating detector. Specifically, if the 3-D object is placed within a chamber that has an opening through which light enters but does not escape, and the wall of the chamber serves as an integrating sphere that converts any photon reaching it into a photoevent, then it is impossible to construct a hologram of the 3-D object using classical means. Quantum holography, permits the making of a hologram of the concealed object by using the signal beam to generate both the probe and the reference, and measuring the idler beam with full spatial resolution, using the time of arrival of the idler photon as a gate. Lacking entanglement, two-photon interferometry by itself cannot achieve this feat.

This work was supported by the National Science Foundation and by the Center for Subsurface Sensing and Imaging Systems (CenSSIS), an NSF engineering research center.

\section{REFERENCES}

1. D. N. Klyshko, Photons and Nonlinear Optics, Gordon and Breach, New York, 1988.

2. A. F. Abouraddy, B. E. A. Saleh, A. V. Sergienko, and M. C. Teich, "Role of entanglement in twophoton imaging," Phys. Rev. Lett. 87, 123602, 2001.

3. A. F. Abouraddy, B. E. A. Saleh, A. V. Sergienko, and M. C. Teich, "Quantum holography," Optics Express, 9, No. 10, 505, 2000.

*besaleh@bu.edu phone:1 (617) 353-7176; fax 1617 353-6440; http://people.bu.edu/besaleh; http://www.bu.edu/qi1; Department of ECE, Boston University, 8 Saint Mary's St., Boston, MA 02215, USA 